\title{
ON THE NONIMMERSION OF PRODUCTS OF REAL PROJECTIVE SPACES
}

\author{
HYUN-JONG SONG AND W. STEPHEN WILSON
}

\begin{abstract}
In this paper we utilize $B P^{*}()$, a generalized cohomology theory associated with the Brown-Peterson spectrum to prove a nonimmersion theorem for products of real projective spaces.
\end{abstract}

\section{Statement of Results}

Let $\nu\left(2^{a}(2 b+1)\right)=a$ and let $\alpha(n)$ denote the number of 1's in the binary expansion of $n$. Let $P^{n}$ denote the $n$-dimensional real projective space. Then our main theorem is

Theorem 1.1. If $d$ and $k$ are positive integers with $\alpha\left(m_{1}\right)+\cdots+\alpha\left(m_{k}\right)=d+k$ and $2^{\nu\left(m_{i}\right)}>d\left(2^{k+1}-1\right)$ for each $i$, then

$$
P^{2 m_{1}+2 d} \times P^{2 m_{2}+4 d} \times \cdots \times P^{2 m_{k}+2^{k} d}
$$

cannot be immersed in

$$
R^{4\left(m_{1}+\cdots+m_{k}\right)-2 d-k-1}
$$

To our knowledge, the only published works on nonimmersions of products of real projective spaces are those of Suzuki [18] and Kobayashi [12]. Their methods seem to be effective for $P^{m} \times P^{n}$ with $m=2^{r}-1$ and $n=2^{s}-1$. When $s>r>7$, their nonimmersions are in dimensions $m+n+2^{s-1}$ (see [12, Corollary 4]). We can apply Theorem 1.1 to the case $m_{1}=2^{r-2}+2^{r-3}$ and $m_{2}=2^{s-2}+2^{s-3}$ when $r-3$ and $s-3$ are greater than 14 and we have $k=2$ and $d=2$. This manifold is a submanifold of theirs and it fails to immerse in dimension $m+n+2^{s-1}+2^{r-1}-5$, which is an improvement of $2^{r-1}-5$ dimensions. A more detailed analysis [17] of this special case lowers the above 14 to 4.

From known immersions of single real projective spaces, e.g., Adem [2], Lam [13], Cohen [7], and Randall [15], we see that nonimmersions in Theorem 1.1

Received by the editors July 18, 1988.

1980 Mathematics Subject Classification (1985 Revision). Primary 57R42, 55N20.

Key words and phrases. Real projective space, $B P^{*}$-theory, Euler class, immersion.

The first author was partially supported by KOSEF.

The second author was partially supported by the N.S.F. 
for the case $k=2$ and $d=1$ (resp. $k=2$ and $d=2$ ) are within 7 (resp. 15) of the best possible.

The case $k=1$ of Theorem 1.1 is the Bendersky-Davis conjecture [5] proven by Davis [9] except that we add the additional hypothesis $2^{\nu(m)}>3(\alpha(m)-1)$. For Davis to prove his theorem he must do a detailed analysis of the BrownPeterson cohomology of the product of two real projective spaces. Our extra hypothesis eliminates the need for this analysis. The combinatorics become quite elementary. The simple proof with this extra hypothesis was done first in [17]. Unfortunately, the extra hypothesis in both the $k=1$ case and the $k>1$ case miss many interesting nonimmersions. Computer calculations done by the second author (using MAPLE on an AT\&T UNIX PC and a SUN3) show that for $k>1$ we cannot completely remove the hypothesis using our approach. It could obviously be weakened significantly but at the cost of a detailed (and difficult) analysis of the Brown-Peterson cohomology of the $k+1$-fold product of real projective spaces. The known descriptions are hopelessly inadequate for more than what we do here [11]. As it is we depend heavily on the Conner-Floyd conjecture [8].

The origin of this paper is in the Ph.D. thesis [17] of the first author. He would like to thank his advisor, Don Davis, for his encouragement and advice. The authors wish to thank Don Davis and Ralph Cohen for helping them get together for this research, and Bill Huggins for help with the computer typesetting.

Mathematically, our debts are clear. We follow Don Davis [9], except that we work with products of projective spaces instead of one. Going back further, this approach was first due to Astey [3]. The hint for the numbers that we find come from the Bendersky-Davis conjecture of [5].

\section{BROWN-PETERSON COHOMOLOGY PRELIMINARIES}

The obstruction to immersion for our $k$-fold product will lie in the 2-primary Brown-Peterson cohomology of the $k+1$-fold product. Brown-Peterson cohomology is generally covered in [1] and also in [19]. $B P^{*} \simeq Z_{(2)}\left[v_{1}, v_{2}, \ldots\right]$ with degree of $v_{n}=-2\left(2^{n}-1\right) . B P^{*} C P^{\infty}$ is the power series over $B P^{*}$ on a 2-dimensional generator $x$, and $B P^{*} C P^{n}$ is a truncated polynomial algebra over $B P^{*}$ with $x^{n+1}=0$. The nontrivial map $P^{2 n} \rightarrow C P^{n}$ is easily seen (by the Atiyah-Hirzebruch spectral sequence) to be surjective in $B P^{*}()$. It also follows that $x$ goes nontrivially to $B P^{2}\left(P^{2 n}\right)$, generates $B P^{*}\left(P^{2 n}\right)$, and in here, $x^{n+1}=0$. The composition

$$
P^{2 n} \rightarrow P^{\infty} \rightarrow C P^{\infty} \stackrel{2}{\rightarrow} C P^{\infty}
$$

is trivial, so the image of $x$ under this map is zero. It is the two-series [2] $(x)$. Thus we have shown

Lemma 2.1. $B P^{*}[x] /\left(x^{n+1},[2](x)\right)$ surjects to $B P^{*}\left(P^{2 n}\right)$. 
In fact it is trivial to show it is an isomorphism, but all we need is the map. We have a map

$$
\begin{gathered}
B P^{*}\left[x_{1}, \ldots, x_{k+1}\right] /\left(x_{1}^{n_{1}+1}, \ldots, x_{k+1}^{n_{k+1}+1},[2]\left(x_{1}\right), \ldots,[2]\left(x_{k+1}\right)\right) \\
\rightarrow B P^{*}\left(P^{2 n_{1}} \times \cdots \times P^{2 n_{k+1}}\right)
\end{gathered}
$$

given by the exterior product.

Lemma 2.2. (a) $2^{d} x_{i}^{n_{i}-j}=0$ if $j<d$.

(b) All monomials in the $x$ 's in degree $>2\left\{n_{1}+\cdots+n_{k+1}-\left(2^{k+1}-1\right)(d+1)\right\}$ are annihilated by $2^{d+1}$.

(c) For $n_{i}>d 2^{i-1}$

$$
2^{d} x_{1}^{n_{1}-d} x_{2}^{n_{2}-2 d} \cdots x_{k+1}^{n_{k+1}-2^{k} d}=v_{k+1}^{d} x_{1}^{n_{1}} x_{2}^{n_{2}} \cdots x_{k+1}^{n_{k+1}} \neq 0 .
$$

Proof. [2] $(x)=2 x+{ }_{F} v_{1} x^{2}+{ }_{F} v_{2} x^{4}+{ }_{F} \cdots$. (Araki generators [19]). Part (a) is an easy induction on $j$. We prove part (b) for any monomial $c$ in the ideal $\left(2, v_{1}, v_{2}, \ldots\right)^{d+1}$ where $c$ times the monomial in the $x$ 's has the stated degree. Consider the element

$$
c x_{1}^{n_{1}-r_{1}} x_{2}^{n_{2}-r_{2}} \cdots x_{k+1}^{n_{k+1}-r_{k+1}}
$$

with $r_{1}+\cdots+r_{k+1}<\left(2^{k+1}-1\right)(d+1)+\operatorname{deg} c$. We define an algorithm to reduce this element. Let $v_{0}=2$. If $i$ is the smallest integer for which $v_{i}$ divides $c$ then replace $v_{i} x_{i+1}^{2^{i}}$ using the corresponding term in $0=[2]\left(x_{i+1}\right)$. Either the power of $x_{i+1}$ increases or a smaller $v_{j}$ shows up. We iterate the process. If $j$ gets down to zero then the next step forces an increase in the power of $x_{1}$. Consequently, this algorithm needs only a finite number of iterations. At the end we are left with only $v_{j}$ 's with $j>k$, and we must still have at least $d+1$ of them. The highest degree element like this is $v_{k+1}^{d+1}$ times the top class. We are in degree greater than this, so our element reduces to zero. Computations of this sort are done in [11, p. 437].

To show (c) we inductively go through the intermediate stages given by

$$
v_{i}^{d} x_{1}^{n_{1}} x_{2}^{n_{2}} \cdots x_{i}^{n_{i}} x_{i+1}^{n_{i+1}-2^{i} d} \cdots x_{k+1}^{n_{k+1}-2^{k} d}
$$

using the easily verified (use the algorithm) fact that

$$
\left(2, v_{1}, \ldots, v_{i-1}\right) x_{1}^{n_{1}} x_{2}^{n_{2}} \cdots x_{i}^{n_{i}}=0 .
$$

To do the inductive step, we use

$$
0=[2]\left(x_{i+1}\right)=\sum^{F} v_{j} x_{i+1}^{2^{j}}
$$

which, $\bmod \left(2, v_{1}, \ldots, v_{i-1}\right)$, is really $0=v_{i} x_{i+1}^{2^{i}}+_{F} v_{i+1} x_{i+1}^{2^{i+1}}+{ }_{F} \cdots$. This gives the next step because $x_{i+1}^{n_{i+1}+1}=0$. 
The tricky part is to show this element is nonzero. This is equivalent to the Conner-Floyd conjecture $[8,16]$. It is enough to show that $v_{k+1}^{n}$ times the top class is nonzero. We have Spanier-Whitehead duality [4]

$$
B P^{*}\left(P^{2 n}\right) \simeq B P_{2^{L-*}}\left(P_{2^{L}-2 n-1}^{2^{L}-1}\right)
$$

for $L$ big. For $N=(k+1)\left(2^{L}-1\right), m_{i}=2^{L}-2 n_{i}-1$, we now have

$$
B P^{*}\left(P^{2 n_{1}} \wedge \cdots \wedge P^{2 n_{k+1}}\right) \simeq B P_{N-*}\left(P_{m_{1}}^{2^{L}-1} \wedge \cdots \wedge P_{m_{k+1}}^{2^{L}-1}\right) .
$$

Our top degree element corresponds to the bottom degree element. The right side maps to $B P_{*}\left(P_{m_{1}}^{\infty} \wedge \cdots \wedge P_{m_{k+1}}^{\infty}\right)$ which is isomorphic, with a degree shift, to $B P_{*}\left(\bigwedge^{k+1} P^{\infty}\right)$ with the bottom class going to the bottom class. The $p=2$ Conner-Floyd conjecture [11, Appendix] following [16] or [14], says that the annihilator ideal of the bottom class is $\left(2, v_{1}, \ldots, v_{k}\right)$, so $v_{k+1}^{n}$ times it is nonzero.

\section{GEOMETRIC PRELIMINARIES}

Let $\xi_{n}$ be the canonical line bundle over $P^{2 n}$. From KO-theory we know that $2^{L} \xi_{n}$ is trivial for $L$ large. It is elementary that $\tau$, the tangent bundle, plus a trivial line bundle $\theta$ is $(2 n+1) \xi_{n}$. The normal bundle $\nu$ is thus $\left(2^{L}-2 n-1\right) \xi_{n} \oplus \theta$. Immersion theory [10] tells us that in our case the geometric dimension of the normal bundle will give us the answer to the immersion question, i.e. an $n$-dimensional projective space with a normal bundle of dimension $K$ has $K-k$ everywhere linearly independent sections if and only if the projective space immerses in dimension $n+k$. This observation, together with the fact that the product of normal bundles gives a normal bundle shows

Proposition 3.1. $P^{2 n_{1}} \times \cdots \times P^{2 n_{k}}$ immerses in $R^{4\left(n_{1}+\cdots+n_{k}\right)-c}$ if and only if

$$
\left(2^{L}-2 n_{1}-1\right) \xi_{2 n_{1}} \times\left(2^{L}-2 n_{2}-1\right) \xi_{2 n_{2}} \times \cdots \times\left(2^{L}-2 n_{k}-1\right) \xi_{2 n_{k}} \oplus k \theta
$$

has $k 2^{L}-\left(4 n_{1}+\cdots+4 n_{k}-c\right)$ linearly independent sections.

We prefer a slightly different bundle, so

Proposition 3.2. If $P^{2 n_{1}} \times \cdots \times P^{2 n_{k}}$ immerses in $R^{4\left(n_{1}+\cdots+n_{k}\right)-c}$ then

$$
\left(2^{L}-2 n_{1}\right) \xi_{2 n_{1}} \times\left(2^{L}-2 n_{2}\right) \xi_{2 n_{2}} \times \cdots \times\left(2^{L}-2 n_{k}\right) \xi_{2 n_{k}}
$$

has $k 2^{L}-\left(4 n_{1}+\cdots+4 n_{k}-c+k\right)$ linearly independent sections.

Proof. Let $\tau_{i}$ be the tangent bundle for $P^{2 n_{i}}$ and $\nu$ the normal bundle of the hypothesized immersion. Then $\left(\tau_{1} \times \cdots \times \tau_{k}\right) \oplus \nu=\left\{4\left(n_{1}+\cdots+n_{k}\right)-c\right\} \theta$. Since $\tau_{i} \oplus \theta=\left(2 n_{i}+1\right) \xi_{2 n_{i}}$ we have

$$
\left\{\left(2 n_{1}+1\right) \xi_{2 n_{1}} \times \cdots \times\left(2 n_{k}+1\right) \xi_{2 n_{k}}\right\} \oplus \nu=\left\{4\left(n_{1}+\cdots+n_{k}\right)-c+k\right\} \theta .
$$

Add

$$
\alpha=\left\{2^{L}-\left(2 n_{1}+1\right)\right\} \xi_{2 n_{1}} \times \cdots \times\left\{2^{L}-\left(2 n_{k}+1\right)\right\} \xi_{2 n_{k}}
$$


to both sides to get

$$
k 2^{L} \theta \oplus \nu=\alpha \oplus\left\{4\left(n_{1}+\cdots+n_{k}\right)-c+k\right\} \theta .
$$

For large $L, k 2^{L}-\left\{4\left(n_{1}+\cdots+n_{k}\right)-c\right\}$ is much bigger than the dimension of our manifold so we can cancel some of the trivial summands to get

$$
\alpha=\left[k 2^{L}-\left\{4\left(n_{1}+\cdots+n_{k}\right)-c+k\right\}\right] \theta \oplus \nu .
$$

The bundle $\alpha$ is a subbundle of our bundle in Proposition 3.2 so there are $k 2^{L}-\left\{4\left(n_{1}+\cdots+n_{k}\right)-c+k\right\}$ sections.

Multiple sectioning problems are reduced to single sectioning problems through the following proposition.

Proposition 3.3 (Becker, [6]). Let $\alpha$ be a real vector bundle over a finite $C W$ complex $X$. If $\alpha$ admits $m+1$ everywhere linearly independent sections over the real numbers then the bundle $\alpha \otimes \xi_{m}$ over $X \times P^{m}$ admits a nowhere zero section.

\section{The Euler Class}

A complex orientable bundle over $X$ has a $B P$ Euler class defined, as usual, by pulling back the Thom class of the bundle. It lies in $B P^{n} X$ where $n$ is the real dimension of the bundle. We collect the elementary facts that we need about the Euler class, $e()$. Let $\eta_{n}$ be the canonical complex line bundle over $C P^{n}$.

Proposition 4.1. Let $\alpha, \beta$ be complex orientable bundles over a finite $C W$ complex $X$.

(a) If $\alpha$ has a nonzero section, then $e(\alpha)=0$.

(b) $e(\alpha \oplus \beta)=e(\alpha) \cup e(\beta)$.

(c) $e\left(\eta_{\infty}\right) \in B P^{2} C P^{\infty}$ can be chosen as the generator.

(d) $e\left(2 \xi_{2 n}\right) \in B P^{2} P^{2 n}$ is the corresponding generator.

We use Astey's reduction:

Proposition 4.2 (Astey [3]).

$$
e\left(2 \xi_{2 n_{i}} \otimes \xi_{2 n_{k+1}}\right)=u\left[e\left(2 \xi_{2 n_{i}}\right)-e\left(2 \xi_{2 n_{k+1}}\right)\right]
$$

where $u$ is a unit in $B P^{*}\left(P^{2 n_{i}} \times P^{2 n_{k+1}}\right)$.

Proposition 4.3. Let $2 n_{i}=2 m_{i}+2^{i} d, i \leq k$. Let

$$
\alpha=\left(2^{L}-2 n_{1}\right) \xi_{2 n_{1}} \times\left(2^{L}-2 n_{2}\right) \xi_{2 n_{2}} \times \cdots \times\left(2^{L}-2 n_{k}\right) \xi_{2 n_{k}} .
$$

If $P^{2 n_{1}} \times \cdots \times P^{2 n_{k}}$ immerses in $R^{4\left(m_{1}+\cdots+m_{k}\right)-2 d-k-1}$ then the Euler class of $\alpha \otimes \xi_{2 n_{k+1}}$ must be zero where $2 n_{k+1}=k 2^{L}-\left[4\left(m_{1}+\cdots+m_{k}\right)-2 d\right]$.

Proof. Use Propositions 3.2, 3.3, and 4.1(a). 


\section{Proof of Theorem 1.1}

It is enough to show the Euler class in Proposition 4.3 is nonzero. $\alpha$ is the sum of pairs of canonical bundles over real projective spaces. We can apply Proposition 4.1(b) and Proposition 4.2 to see that the nontriviality of the Euler class is equivalent to the nontriviality of (recall from Proposition 4.1(d) that $\left.x_{i}=e\left(2 \xi_{2 n_{i}}\right)\right)$

$$
\prod_{1}^{k}\left[x_{i}-x_{k+1}\right]^{L^{L-1}-n_{i}}
$$

which is in degree $2\left(n_{1}+\cdots+n_{k+1}\right)-2\left(2^{k+1}-1\right) d$, the degree of $v_{k+1}^{d}$ times the top class. This is

$$
\sum_{j_{1}, \ldots, j_{k}} \pm\left(\begin{array}{c}
2^{L-1}-n_{1} \\
n_{1}-j_{1}
\end{array}\right) \cdots\left(\begin{array}{c}
2^{L-1}-n_{k} \\
n_{k}-j_{k}
\end{array}\right) x_{1}^{n_{1}-j_{1}} \cdots x_{k}^{n_{k}-j_{k}} x_{k+1}^{n_{k+1}+j_{1}+\cdots+j_{k}-d\left(2^{k+1}-1\right)} \text {. }
$$

We need to deal with the binomial coefficients.

Proposition 5.1. If $n=m+s$ with $2^{\nu(m)}>s$, then for $L$ sufficiently large, we have

$$
\begin{array}{rlrl}
\nu\left(\begin{array}{c}
2^{L}-n \\
n-j
\end{array}\right) & \geq \alpha(m) & & \text { if } 0 \leq j<2 s, \\
& \geq \alpha(m)-1 & & \text { if } j=2 s, \\
& \text { if } 2 s<j<2^{\nu(m)} .
\end{array}
$$

Note that our hypothesis implies $\alpha(n)=\alpha(m)+\alpha(s)$.

Proof. Using the identity

$$
\nu\left(\begin{array}{l}
a \\
b
\end{array}\right)=\alpha(b)+\alpha(a-b)-\alpha(a)
$$

and the relations

$$
\begin{aligned}
\alpha\left(2^{L}-m-s\right) & =\alpha\left(2^{L}-2^{\nu(m)}-m-s+2^{\nu(m)}\right) \\
& =\alpha\left(2^{L}-2^{\nu(m)}-m\right)+\alpha\left(2^{\nu(m)}-s\right) \\
& =\alpha\left(2^{L}-2^{\nu(m)+1}-2 m\right)+1+\alpha\left(2^{\nu(m)}-s\right) \\
& =\alpha\left(2^{L}-2^{\nu(m)+1}-2 m\right)+\alpha\left(2^{\nu(m)+1}-s\right)
\end{aligned}
$$

and

$$
\alpha\left(2^{L}-2 m-2 s+j\right)=\alpha\left(2^{L}-2^{\nu(m)+1}-2 m\right)+\alpha\left(2^{\nu(m)+1}-2 s+j\right)
$$

we have that

$$
\begin{aligned}
\nu\left(\begin{array}{c}
2^{L}-n \\
n-j
\end{array}\right) & =\alpha(m+s-j)+\alpha\left(2^{L}-2 m-2 s+j\right)-\alpha\left(2^{L}-m-s\right) \\
& =\alpha(m+s-j)+\alpha\left(2^{\nu(m)+1}-2 s+j\right)-\alpha\left(2^{\nu(m)+1}-s\right) .
\end{aligned}
$$

If $0 \leq j \leq s$ then $\alpha(m+s-j)=\alpha(m)+\alpha(s-j)$ so the above is equal to

$$
\nu\left(\begin{array}{c}
2^{\nu(m)+1}-s \\
s-j
\end{array}\right)+\alpha(m) \geq \alpha(m) .
$$


If $s<j<2^{\nu(m)}$ then $\alpha(m+s-j)=\alpha\left(m-2^{\nu(m)}\right)+\alpha\left(2^{\nu(m)}+s-j\right)$ so the above is equal to

$$
\alpha\left(2^{\nu(m)+1}-2 s+j\right)-\alpha\left(2^{\nu(m)+1}-s\right)+\alpha\left(m-2^{\nu(m)}\right)+\alpha\left(2^{\nu(m)}+s-j\right) .
$$

If $s<j<2 s$ then this is

$$
1+\alpha\left(2^{\nu(m)}-2 s+j\right)-\alpha\left(2^{\nu(m)+1}-s\right)+\alpha\left(m-2^{\nu(m)}\right)+\alpha\left(2^{\nu(m)}+s-j\right)
$$

which is

$$
\begin{gathered}
\alpha\left(2^{\nu(m)}-2 s+j\right)-\alpha\left(2^{\nu(m)+1}-s\right)+\alpha\left(2^{\nu(m)}+s-j\right)+\alpha(m) \\
=\nu\left(\begin{array}{c}
2^{\nu(m)+1}-s \\
2^{\nu(m)}+s-j
\end{array}\right)+\alpha(m) \geq \alpha(m) .
\end{gathered}
$$

If $j=2 s$ then this is equal to

$$
\begin{aligned}
& \alpha\left(2^{\nu(m)+1}\right)-\alpha\left(2^{\nu(m)+1}-s\right)+\alpha\left(m-2^{\nu(m)}\right)+\alpha\left(2^{\nu(m)}-s\right) \\
& \quad=1-\alpha\left(2^{\nu(m)+1}-s\right)+\alpha\left(m-2^{\nu(m)}\right)+\alpha\left(2^{\nu(m)+1}-s\right)-1 \\
& =\alpha\left(m-2^{\nu(m)}\right)=\alpha(m)-1 .
\end{aligned}
$$

Otherwise, if $2 s<j<2^{\nu(m)}$ then

$$
\begin{aligned}
& \alpha\left(2^{\nu(m)+1}-2 s+j\right)-\alpha\left(2^{\nu(m)+1}-s\right)+\alpha\left(m-2^{\nu(m)}\right)+\alpha\left(2^{\nu(m)}+s-j\right) \\
& \quad=\alpha\left(2^{\nu(m)}-2 s+j\right)-\alpha\left(2^{\nu(m)+1}-s\right)+\alpha\left(m-2^{\nu(m)}\right)+\alpha\left(2^{\nu(m)}+s-j\right) \\
& \quad=\nu\left(\begin{array}{c}
2^{\nu(m)+1}-s \\
2^{\nu(m)}+s-j
\end{array}\right)+\alpha\left(m-2^{\nu(m)}\right) \\
& \quad=\nu\left(\begin{array}{c}
2^{\nu(m)+1}-s \\
2^{\nu(m)}+s-j
\end{array}\right)+\alpha(m)-1 \geq \alpha(m)-1 .
\end{aligned}
$$

Define

$$
A_{i, j} \equiv\left(\begin{array}{c}
2^{L-1}-n_{i} \\
n_{i}-j
\end{array}\right) .
$$

A typical term in our Euler class expansion has coefficient

$$
\pm A_{1, j_{1}} \cdots A_{k, j_{k}} \cdot
$$

By Proposition 5.1 and the fact that $j_{i} \leq d\left(2^{k+1}-1\right)<2^{\nu\left(m_{i}\right)}$ we have

$$
\nu\left(A_{1, j_{1}} \cdots A_{k, j_{k}}\right) \geq \alpha\left(m_{1}\right)+\cdots+\alpha\left(m_{k}\right)-k=d .
$$

However, if $j_{t}>2^{t} d$ for some $t$, then $j_{i}<2^{i} d$ for some $i$ and $\nu\left(A_{1, j_{1}} \cdots A_{k, j_{k}}\right)$ $>d$ and by Lemma 2.2(b) the term gives zero. So all terms give zero except the one with $j_{i}=2^{i} d$ for all $i$, where $\nu\left(A_{1, j_{1}} \cdots A_{k, j_{k}}\right)=d$ and by Lemma 2.2(c) we get a nonzero Euler class. This concludes the proof of Theorem 1.1. 


\section{REFERENCES}

1. J. F. Adams, Quillen's work on formal groups and complex cobordism, Stable Homotopy and Generalized Homology, Univ. of Chicago Press, Chicago, 1974, pp. 29-120.

2. J. Adem, Some immersions associated with bilinear maps, Bol. Soc. Mat. Mexicana 13 (1968), 95-104.

3. L. Astey, Geometric dimension of bundles over real projective spaces, Quart. J. Math. Oxford 31 (1980), 129-155.

4. M. F. Atiyah, Thom complexes, Proc. London Math. Soc. (3) 11 (1961), 291-310.

5. M. Bendersky and D. M. Davis, Unstable BP-homology and desuspensions, Amer. J. Math. (4) 107 (1985), 833-852.

6. J. C. Becker, On the existence of $A_{k}$-structure on stable vector bundles, Topology 9 (1970), 367-384.

7. R. Cohen, The immersion conjecture for differentiable manifolds, Ann. of Math. 121 (1985), 237-328.

8. P. E. Conner and E. E. Floyd, Differential periodic maps, Springer, Berlin, 1964.

9. D. M. Davis, A strong non-immersion theorem for real projective spaces, Ann. of Math. $\mathbf{1 2 0}$ (1984), 517-528.

10. M. W. Hirsch, Immersions of manifolds, Trans. Amer. Math. Soc. 93 (1959), 242-276.

11. D. C. Johnson and W. S. Wilson, The Brown-Peterson homology of elementary p-groups, Amer. J. Math. 107 (1985), 427-453.

12. T. Kobayashi, Note on $\gamma$-dimension and products of real projective spaces, J. Math. Soc. Japan 34 (1982), 501-505.

13. K. Y. Lam, Construction of non-singular bilinear maps, Topology 6 (1967), 423-426.

14. S. A. Mitchell, A proof of the Conner-Floyd conjecture, Amer. J. Math. 102 (1980), 889-902.

15. A. D. Randall, Some immersion theorems for projective spaces, Trans. Amer. Math. Soc. 147 (1970), 135-151.

16. D. C. Ravenel and W. S. Wilson, The Morava K-theories of Eilenberg-MacLane spaces and the Conner-Floyd conjecture, Amer. J. Math. 102 (1980), 691-748.

17. H.-J. Song, On the non-immersion of products of real projective spaces, Ph.D. Thesis, Lehigh Univ., 1987.

18. H. Suzuki, Operations in KO-theory and products of real projective spaces, Mem. Fac. Sci. Kyushu Univ. Ser. A 18 (1964), 140-153.

19. W. S. Wilson, $A$ BP introduction and sampler, CBMS Regional Conf. Series in Math., no. 48, Amer. Math. Soc., Providence, R.I., 1982.

Department of Applied Mathematics, National Fisheries University of Pusan, Pusan 608, KOREA

Department of Mathematics, The Johns Hopkins University, Baltimore, Maryland 21218 